dans un consensus qui rassemble finalement Albert Soboul et Jean Tulard, sur un Napoléon, sauveur des intérêts de la bourgeoisie. Le dictateur clôt la Révolution, dont il préserve une partie des acquis tout en établissant un despotisme militaire et en se lançant dans la surenchère guerrière à l'extérieur. Son action européenne consiste surtout à vassaliser le reste du continent à son Empire, sans théorie construite d'avance, et a suscité en réaction la naissance des « nationalismes du ressentiment $\gg$.

La troisième et dernière partie fait le bilan des débats scientifiques actuels. L'Empire est davantage envisagé dans ses aspects législatifs et administratifs, économiques et sociaux. Louis Bergeron à l'École des hautes études en sciences sociales, initiateur de l'étude des « masses de granit », mais aussi François Crouzet ou Denis Woronoff, pour ne citer qu'eux, ont été les artisans de cet effort de recherche. Petiteau, dans des développements stimulants, fait le bilan des réponses apportées par ces travaux, et rend compte des renouvellements actuels de la recherche. L'histoire sociale y conserve un rôle pionnier, avec des thèses de référence, comme celle de Claude-Isabelle Brelot, à qui ce livre est dédié, sur la noblesse franc-comtoise. On se reportera à la bibliographie qui, sans prétendre à l'exhaustivité, livre une appréciable mise à jour des ouvrages et articles sur les thèmes de recherche récents.

L'ouvrage de Natalie Petiteau comporte donc un intérêt double. À l'analyse de la construction lente et longtemps contrariée d'une histoire objective, dégagée des légendes partisanes liées à la figure de Napoléon, s'ajoute une synthèse utile sur la recherche en cours. Relayée désormais par les manuels scolaires, la disqualification de la mythologie napoléonienne par l'historien n'empêche pourtant pas celle-ci de demeurer présente dans la mémoire nationale, par le biais d'une littérature qui fleurit de commémoration en bicentenaire.

Nicolas LAUNOIS

\title{
ARCHIVES ET HISTOIRE INTELLECTUELLE
}

Francis JoAnnès, dir., Rendre la justice en Mésopotamie. Archives judiciaires du Proche-Orient ancien (III ${ }^{e-} I^{e r}$ millénaire avant J.-C.). Saint-Denis, Presses universitaires de Vincennes, 2000. $14 \times 22,272$ p., bibliogr., glossaire, index (Temps \& espaces).

Le titre de cet ouvrage rend compte des deux principales démarches suivies par les auteurs : d'une part, répondre à un projet de vision globale de la justice en 
Mésopotamie, et d'autre part, appuyer ce projet sur une étude détaillée de plusieurs sources documentaires.

Ces sources, des tablettes en argile couvertes de signes cunéiformes et datées du $\mathrm{III}^{\mathrm{e}}$ au I ${ }^{\mathrm{er}}$ millénaire avant J.-C., fournissent depuis leurs premières exhumations au $\mathrm{XIX}^{\mathrm{e}}$ siècle, une abondante documentation concernant les pratiques de la justice et du droit. Par exemple, Adam Falkenstein publie et étudie, en 1957, environ deux cent cinquante de ces tablettes. Son travail colossal permet de mieux comprendre les mécanismes judiciaires, mais le contexte historique même n'est guère pris en considération. Puis, la spécialisation croissante des études assyriologiques et la nécessité d'une rigueur philologique de plus en plus accentuée ont morcelé progressivement le domaine de la recherche sur le Proche-Orient ancien. Cette diversité dans le monde assyriologique correspond également à une caractéristique majeure de la société mésopotamienne : elle est le fruit de confrontations, pacifiques ou non, de fusions et de transformations de plusieurs groupes sociaux et culturels. Il faut ajouter à ce paysage une dimension temporelle (les premières traces d'urbanisation remontent au $\mathrm{IV}^{\mathrm{e}}$ millénaire), et géographique (la région concernée est recouverte actuellement par l'Irak, l'Iran et une partie de la Syrie au nord), et nous obtenons ainsi une mosaïque riche et diversifiée de pratiques et de cultures. Malgré cette hétérogénéité, certains points communs apparaissent dans la civilisation mésopotamienne durant trois millénaires d'histoire. Parmi eux, on peut citer l'usage général de l'écriture cunéiforme et les tentatives de plus en plus forte de centralisation et de contrôle du pouvoir politique. Il existe donc, selon l'expression de Bertrand Lafont, « une tension entre unité et diversité dans l'histoire ancienne de la Mésopotamie ».

Malgré ce paradoxe, le lecteur néophyte, familiarisé peut-être seulement aux noms de «sumérien » et d' « akkadien », n'est nullement dérouté en commençant la lecture de cet ouvrage. Cela correspond au premier souci des auteurs : rendre accessibles certains traits principaux de la civilisation mésopotamienne. À cette fin, la chronologie présentée par Bertrand Lafont dès le début de l'ouvrage, ainsi que la carte géographique offrent un panorama succinct et très utile.

Un second souci est à l'origine de la structure même de l'ouvrage. Francis Joannès n'a pas voulu réunir, comme il le précise, les travaux de plusieurs assyriologues, afin de présenter des sources exhaustives puis d'en tirer quelques conclusions. Son objectif est de proposer un « éclairage » des pratiques judiciaires mésopotamiennes, aux dimensions profondément humaines.

Une synthèse de ces pratiques est donc présentée dès le premier chapitre par Sophie Lafont, et répond aux exigences citées précédemment : familiariser rapidement le lecteur aux procédures et institutions judiciaires en Mésopotamie, fournir un vaste aperçu directement utilisable - par exemple, par des historiens du droit. Pour cela, il est nécessaire de briser quelques idées reçues. Ainsi, même si le roi se revendique couramment comme source même de toute justice, il n'use pas d'un autoritarisme abusif comme le laisse entendre le mythe occidental du « despotisme oriental ». Il existe, en effet, des juridictions locales, même si elles ont été progressivement absorbées dans l'appareil étatique. Le roi leur déléguait une certaine 
autorité judiciaire. Sophie Lafont distingue dans ce processus la justice « retenue » de la justice « déléguée ».

Il n'est souvent pas facile de décrire clairement le cadre humain, temporel et spatial de ces institutions judiciaires. Mais il est certain que rendre la justice n'est pas le seul apanage des juges. Des officiers administratifs ou des professionnels dans des domaines requérant une compétence spécifique, comme le droit commercial, peuvent se voir attribuer des fonctions judiciaires. S'il ne semble pas exister d'avocats, de véritables auxiliaires de justice sont attestés, jouant un rôle proche de l'huissier ou de l'avoué moderne. Enfin, le roi lui-même, intervenant ex officio dans tous les procès, se préoccupe essentiellement de trancher sur des affaires politiques et de complots. Il n'existe en revanche pas de trace de hiérarchie judiciaire. De même, aucun bâtiment spécifique tenant lieu de tribunal n'est attesté. Les juridictions sont réunies temporairement, même si certains procès durent plusieurs années, et traduisent par leur composition hétérogène - officiers administratifs, juges... - une absence de séparation des pouvoirs. Ce trait ne doit pourtant pas conduire à une vision autoritaire et monolithique de la justice. Plusieurs points particuliers, dans son fonctionnement, révèlent des soucis d'équité. Ainsi le roi est-il en théorie accessible pour tout plaideur - même prisonnier évadé - insatisfait des compétences d'une juridiction locale. La capacité d'ester est également ouverte aux femmes, aux esclaves et aux mineurs représentés par un tuteur. Ces juridictions sont de plus laïques; il n'existe aucun tribunal ecclésiastique. Une volonté d'objectivité dans les jugements s'exprime par la considération très fréquente de témoignages oraux et de preuves écrites. Le recours aux serments prêtés par les différentes parties devant les autorités divines ou la pratique de l'ordalie permettent de trancher éventuellement en cas d'incompatibilité de ces témoignages. Ces divers aspects brisent les derniers a priori d'une justice mésopotamienne statique et arbitraire.

Les chapitres suivants se succèdent dans un ordre chronologique. Ils sont organisés selon le même modèle : après une brève description du contexte historique, social et politique du lieu d'origine des sources, chaque chercheur présente les documents qu'il a sélectionnés. Un résumé sur leur contenu et leur portée précède leur traduction. L'évocation fréquente de termes ou d'expressions sumériennes et akkadiennes rend compte des bases philologiques rigoureuses à l'étude des textes, et souligne parfois certaines difficultés d'interprétation.

Dans le deuxième chapitre, Bertrand Lafont présente des textes judiciaires sumériens, documents les plus anciens de cet ouvrage. La distinction entre les procédures civiles et pénales est implicitement présente dans les sources en étant associée au mode même de procédure. Bertrand Lafont classifie donc ces documents d'après leur caractère relevant plutôt du droit privé - mariage et famille, héritages et successions, contentieux à la suite de contrats... - ou plutôt du droit pénal crimes et délits...

Dominique Charpin reprend en partie cette classification pour les textes paléobabyloniens dans le chapitre suivant, et discute plus particulièrement du célèbre code du roi babylonien Hammurabi au XVIII ${ }^{\mathrm{e}}$ siècle avant J.-C. Cet exemple met en évidence les difficultés d'interprétation des textes par des historiens du droit et des 
philologues. Est-ce un recueil législatif ? Ou bien est-ce une inscription commémorative en l'honneur du roi et de ces décisions en matière de justice, destinée aux générations futures? Le débat reste actuellement ouvert chez les assyriologues. Néanmoins, pour Charpin, ce texte est avant tout le produit d'une longue tradition séculaire et de pratiques judiciaires propres à Hammurabi. Cette dimension, à la fois synchronique et diachronique, caractérise de façon générale l'évolution de l'application de la justice en Mésopotamie. Chaque système social et organisation politique nouvellement constitués se réapproprient les pratiques antérieures, les adaptent, les abandonnent ou les modifient, selon des exigences propres.

Ainsi l'augmentation des échanges commerciaux avec l'Asie mineure à l'époque paléo-assyrienne, entraîne-t-elle l'application nécessaire d'un droit commercial évolutif. C'est le sujet du chapitre IV, dans lequel Cécile Michel met en relief, à travers des lettres, des contrats et des procès verbaux de jugement, certains litiges rendant caducs les accords moraux entre différents partenaires. Dans le chapitre v, Brigitte Lion décrit des comptes rendus de procès, issus d'archives privées soigneusement conservés par la partie gagnante - dans le royaume d'Arrapha au $\mathrm{XIV}^{\mathrm{e}}$ siècle avant J.-C. Les sources d'Ugarit du XIV ${ }^{\mathrm{e}}$ au XII ${ }^{\mathrm{e}}$ siècle, examinées par Sylvie Lackenbacher dans le chapitre VI, ont un caractère différent des précédentes, en raison de leur nombre très faible, mais aussi de leur nature : elles concernent des actes royaux au sujet de propriétés foncières. Ce ne sont pas des procès-verbaux, mais des titres de propriété remis au gagnant du procès par le roi.

Dans le chapitre VII, Pierre Villard souligne les difficultés typologiques rencontrées pour essayer de classer des documents judiciaires néo-assyriens du début du $\mathrm{I}^{\mathrm{er}}$ millénaire avant J.-C., conservés aussi bien dans des archives familiales, que par les autorités judiciaires de cette période.

Joannès répond, à travers sa classification des tablettes néo-babyloniennes du dernier chapitre, à son objectif de mise en relief des réalités de la vie quotidienne en Mésopotamie. Cet objectif est atteint, sur l'ensemble de l'ouvrage, de façon convaincante. Les démarches rencontrées dépassent le simple cadre d'une histoire de la justice. Les accents particulièrement humains dans la réaction de ce plaideur victime de vol, dans la prétention à la liberté de cet esclave, dans cette mauvaise foi évidente entre ces deux commerçants, ne figent pas la civilisation mésopotamienne dans de simples traces écrites immortalisées par le temps. Ce type d'étude fournit à la fois un éclairage synthétique, qui ne se veut pas exhaustif, et une mise en lumière particulière des différents aspects de la société à travers l'exposé des documents, utile et nécessaire pour contribuer à une véritable histoire sociale des pratiques judiciaires. On peut regretter parfois le manque de critères explicites dans le choix même des sources. Mais il est difficile de faire face à une documentation lacunaire ou, au contraire, à un lot très abondant de tablettes. Certains auteurs ont donc sélectionné un échantillonnage de textes pour répondre à l'objectif « d'éclairage » sur la société. Une explication parfois plus détaillée sur la pertinence et le choix de certaines sources pourrait compléter cet objectif. Mais ce détail n'enlève rien au grand mérite de l'ouvrage : dévoiler à un public de plus en plus vaste, composé ou non de chercheurs, des pratiques de la société mésopotamienne en ouvrant ainsi le 
champ à des travaux interdisciplinaires. On ne peut que souhaiter le développement de ce type d'étude dans d'autres domaines, comme celui des différentes sciences.

Grégory CHAMBON

Frank Hieronymus, 1488 Petri/Schwabe 1988. Eine traditionsreiche Basler Offizin im Spiegel ihrer frühen Drucke. Vol. I et II. Bâle, Schwabe, 1997. 22,4×30,5, 6-XXII-138-16 + 854 p. et VI-1016 p., ill. n. et coul., index, bibliogr.

Ce monumental catalogue d'exposition est en même temps un ouvrage commémoratif, destiné à fêter le demi-millénaire d'une maison d'édition bâloise qui, pour avoir changé de main (et de nom) à de nombreuses reprises, a cependant connu depuis sa création une activité ininterrompue. L'exposition eut lieu à Bâle en 1988 : la rédaction du catalogue a pris près de dix ans. Au vu du résultat, on en vient presque à trouver ce délai assez court.

Sur un total de près de 2000 pages, Frank Hieronymus a décrit 647 imprimés parus chez les éditeurs de la dynastie Petri en l'espace de deux siècles, entre 1496 et 1693. Ce n'est là (précise-t-il p. E60b) qu'une bonne moitié de la production connue des Petri et Henricpetri à Bâle ; la firme dans son ensemble peut se targuer, jusqu'en 1800, de quelque 3750 imprimés.

Une longue introduction, nourrie de documents d'archives, retrace l'histoire de la maison. Son fondateur, Johannes Petri (1441-1511), qui devient bourgeois de Bâle en 1488, est qualifié d'imprimeur (Buchdrucker) dès la première mention de son nom dans le registre de la ville. Associé de Johannes Amerbach, sans doute en tant que prote, il devient dès 1488 imprimeur autonome, l'égal d'Amerbach, tout comme son collègue Johannes Froben. À eux trois, les Johannes tres, comme ils se désignent eux-mêmes sur la page de titre de leur édition collective - la première - des œuvres de saint Augustin (1506), vont entretenir des années durant une fructueuse collaboration, sous la direction scientifique d'Amerbach.

Le neveu de Johannes Petri, Adam Petri (1454-1527), entre, lui, de plain-pied dans les luttes religieuses en réimprimant notamment nombre d'écrits de Luther, ainsi que la Causa boemica de Jean Hus en 1520. Cet ouvrage avait paru quelques mois plus tôt. Adam Petri le réimprime sans nom d'auteur, sans nom d'éditeur et sans indication de lieu, sous le titre agressif de Liber egregius de unitate ecclesia, cujus autor periit in concilio Constantiensi. Cette édition sera connue de Luther, de Zwingli, de Beatus Rhenanus, qui la feront connaître à leur tour autour d'eux.

Après la mort d'Adam Petri, c'est son fils cadet Heinrich (1508-1579) qui poursuit l'entreprise. Cinquante années durant, Heinrich Petri couvrira dans son activité tous les champs du savoir. L'événement décisif de sa carrière d'imprimeur est le remariage de sa mère avec le mathématicien et cosmographe hébraïsant Sébastien Munster, vers 1529-1530. Dès lors les publications de Munster chez Heinrich Petri feront de ce dernier l'un des principaux imprimeurs du temps en matière de langue hébraïque et de cartes géographiques. 
En 1556, Heinrich Petri, par l'intermédiaire de Vésale, alors médecin impérial, brigue son anoblissement auprès de Charles Quint. Plaidant en sa faveur, Vésale souligne que Petri n'a jamais imprimé un seul livre de Luther. Selon Hieronymus, cette attitude pourrait correspondre davantage à une conviction intérieure qu'à un calcul opportuniste. Il se peut en effet que Petri ait appartenu à une catégorie de chrétiens assez peu attachés au catholicisme traditionnel, mais que les nouveautés de la Réforme ne séduisaient pas davantage. Petri fut, en tout cas, député de la ville dans les questions religieuses, ce qui l'amena par exemple à prendre part, en 1558-1559, au procès de l'hérétique David Joris.

À partir de 1565, les publications de Heinrich Petri paraissent sous la marque de l'Officina Henricpetrina. Ses fils Sixtus, Sebastian et Heinrich Adam semblent avoir adopté eux-mêmes, vers cette époque, le patronyme Henricpetri, peut-être en conséquence de l'anoblissement de leur père. C'est Sebastian Henricpetri (1546-1627) qui, à la mort de son père (1579), poursuit l'entreprise sous son propre nom.

On ne peut ici poursuivre longuement et en détail cette saga qui se prolonge à travers les XVII ${ }^{\mathrm{e}}$ et XVIII ${ }^{\mathrm{e}}$ siècles pour nous mener jusqu'à l'achat de la firme en 1868 par Benno Schwabe et, de là, jusqu'à nos jours. Disons seulement que l'introduction de l'ouvrage, qui comporte des tableaux généalogiques et un index, hélas très incomplet, est enrichie de nombre de reproductions, parmi lesquelles celle d'un placard imprimé en 1784 donnant la liste alphabétique des imprimeurs bâlois depuis les origines de l'imprimerie. Dans un long développement sur les marques d'imprimeurs successivement utilisées, se trouve - outre le cliché d'une lettre autographe de Vésale à Heinrich Petri - la reproduction en couleurs de la lettre d'anoblissement de Charles Quint comprenant en son centre les armes accordées à Petri, c'est-à-dire le roc enflammé et frappé d'un marteau originellement dessiné en 1527, sans doute par Hans Holbein, pour le père de Heinrich, Adam Petri, d'après un verset de Jérémie (Jér. 23, 29). Plusieurs catalogues de livres sont également reproduits, notamment celui de 1576 des ouvrages publiés par l'Officina Henricpetrina et celui de 1578 des livres parus chez Sebastian Henricpetri. On trouve, enfin, reproduits sur près de cinquante pages toute une série de documents portant sur l'histoire de la firme de 1768 à 1877.

Vient ensuite le catalogue proprement dit. Les notices qui le composent sont à la fois classées chronologiquement et groupées par auteurs. Tous les ouvrages décrits sont conservés à la Bibliothèque publique et universitaire de la ville ; la cote et la provenance sont toujours indiquées.

Les reproductions en pleine page sont légion. D'excellente qualité, souvent spectaculaires, comme celles d'un évangéliaire imprimé en 1514 ( $\left.\mathrm{n}^{\circ} 30\right)$, elles font de cet ouvrage un livre d'initiation extrêmement complet à l'art de l'imprimerie aux $\mathrm{XVI}^{\mathrm{e}}$ et $\mathrm{XVII}^{\mathrm{e}}$ siècles. En outre, elles permettent plus d'une fois de fructueuses comparaisons entre l'imprimé et le manuscrit qui lui a servi de base. Quant aux commentaires, ils se présentent comme une histoire en continu d'une grande part de la production éditoriale de la firme.

D'un intérêt tout particulier - mais ce n'est là qu'un exemple parmi d'autres sont les trois notices successivement consacrées à la Margarita philosophica de Gregor Reisch ( ${ }^{\circ}$ 63-65) : une édition de 1517 est suivie de celle procurée par 
Oronce Finé en 1535, considérablement augmentée et, quant aux illustrations, redessinée pour une grande part vraisemblablement par Finé lui-même ; la troisième notice porte sur une réédition de 1583 de l'édition de Finé. Grâce aux illustrations généreusement dispensées sur près de trente pages, on peut mesurer l'écart qui existe entre ces diverses rééditions.

D'autres points sont remarquables : les reproductions en rouge et noir tirées de la Polygraphia de Johannes Trithemius (1518) $\left(\mathrm{n}^{\circ} 66\right)$; les gracieuses planches accompagnant le poème satirique de Thomas Murner, Die Geuchmat zu straff allen wybschen mannen (1519) $\left(\mathrm{n}^{\circ} 73\right)$; la reproduction intégrale des pièces liminaires de la traduction allemande, due à Martin Bucer, de l'édition commentée par Johannes Bugenhagen des Psaumes (1526) (n 134b, p. 363-381) : il s'agit successivement des préfaces de Bucer, de Luther, de Melanchton, de Bugenhagen et à nouveau de Luther et de Bucer.

Mais c'est évidemment à Sébastien Munster que revient dans ce catalogue la part du lion, avec sa Compositio horologiorum de 1531, ses éditions en hébreu, ses cartes et sa Cosmographia. Étendue sur plus de trois cents pages (p. 448-764), la richesse de l'illustration et des notices défie ici la description. De plus, la musique trouve également son compte dans ce catalogue, avec des reproductions de gammes extraites du $\Delta \omega \delta \varepsilon \varkappa \alpha ́ \chi \chi \rho \delta o v$ de Glarean (1547) ( $\left.\mathrm{n}^{\circ} 309\right)$.

Dans le second volume défilent tous les grands noms de la Renaissance italienne, Jérôme Cardan en tête : de généreux extraits de ses œuvres (p. 1014-1059) s'ajoutent à un extrait substantiel de l'introduction qui lui est consacré (p. E23-E28). Très remarquables du point de vue iconographique sont les portraits attribués à Tobias Stimmer, qui ornent à partir de 1575 les éditions des Elogia de Paolo Giovio publiées conjointement par Heinrich Petri et Pietro Perna ( $n^{\circ} 403-404$, p. 1147-1174). Parmi les portraits reproduits (tous furent copiés par Tobias Stimmer à Como, dans la propre galerie de Paolo Giovio) se trouvent celui de Giovio lui-même, mais aussi ceux de Robert, roi de Naples, de l'empereur Maximilien, du pape Léon X et du sultan Osman Irr (présenté par erreur comme Soliman Irr), ceux de Dante, de Pétrarque, de Théodore Gaza et de Jean Lascaris, de Rodolphe Agricola, de Jean Pic de La Mirandole, de Savonarole et de Pomponazzi.

L'ouvrage ne comporte pas moins de neuf index. Le premier d'entre eux, qui est une liste chronologique des éditions présentées dans le catalogue, permet de rétablir l'ordre exact de leur succession dans le temps. Viennent ensuite un index des auteurs et ouvrages anonymes ; un index des imprimeurs, des libraires et des lieux d'impression ; un index des éditeurs scientifiques, des traducteurs et des auteurs de dédicaces, de préfaces et de pièces liminaires ; un index des dédicataires (qui est, faut-il le dire, du plus grand intérêt pour l'étude du mécénat lettré à la Renaissance) ; un index des illustrateurs et des relieurs ; un index des marques d'imprimeurs des maisons Petri et Henricpetri ; un index des anciens possesseurs des exemplaires décrits dans le catalogue ; enfin, un index général des noms de personnes, de lieux et des matières, qui permet aussi bien de repérer, par exemple, les ouvrages d'astrologie et d'astronomie que les manuels scolaires (Schulbuch), les ouvrages en hébreu, les commentaires ou les nombreux manuscrits évoqués - bien sûr, dans les 
limites propres à tout classement par matières. Néanmoins, Montaigne (p. 1820a) n'a aucune raison de figurer sous l'entrée «De Montaigne », et Karel Žerotín (p. 1811a) se trouve par erreur à l'entrée « Karl von Zerotin » pour une occurrence, tandis que, pour les autres, on le trouve correctement à l'entrée «Zerotin ».

Comme l'a écrit Hieronymus à la fin de son introduction (p. E72), « chaque lecteur trouvera dans ce catalogue non seulement des fautes, mais de nombreuses lacunes dans son propre domaine », ce qui s'explique sans peine par la difficulté de maîtriser l'immense variété des domaines couverts par l'activité éditoriale de la dynastie des Petri. Ce n'est donc pas dénigrer ce livre, mais au contraire rendre hommage à l'érudition et à l'honnêteté de l'auteur, que de rectifier ici quelques points erronés découverts au hasard de la consultation. À propos de Pomponazzi, l'auteur n'a pas compris le nom du dédicataire de l'édition de 1567 (p. 1070 et 1071, n. 3) : ce Vidamus de Chartres n'est pas le Guido Vidame, éditeur de textes d'astrologie, que Hieronymus mentionne d'après Jöcher, mais Jean de Ferrières, vidame de Chartres (du latin vice dominus), un des principaux chefs du parti calviniste en France, dont Léon de Bastard d'Estang rédigea la biographie en 1858. En outre, Hieronymus ne connaît pas l'article de Manuela Doni, «Il De incantationibus di Pietro Pomponazzi e l'edizione di Guglielmo Grataroli » (Rinascimento, seconda serie, 15, 1975, p. 183-230), qui montre que Gratarolo, calviniste convaincu, apporta au traité de Pomponazzi des modifications conformes à ses propres convictions religieuses, ce dont on ne peut se douter qu'en comparant son édition - qui sert si souvent de référence - avec les manuscrits. De fait, Hieronymus ne connaît guère Gratarolo lui-même (quoiqu'il n'ignore pas les travaux fondateurs de Carlos Gilly sur Theodor Zwinger), sinon il n'eût pas commis l'erreur (p. 1196a-b) d'attribuer à ce médecin, favorable à l'alchimie mais hostile à Paracelse, un dialogue anonyme intitulé Chrysorrhoas, tout à la gloire de Paracelse ; Gratarolo ne fut d'ailleurs pas le premier à le publier : la première édition, déjà anonyme, était parue à Cologne chez Maternus Cholin en 1559, ce qui suffit à écarter le soupçon que Gratarolo en ait été l'auteur. En outre, Hieronymus n'a pas su (p. 1197b) que l'édition de 1572 du De consideratione quintoe essentice de Johannes de Rupescissa n'était que la seconde : la première avait paru, dès 1561, publiée par le même Gratarolo chez les mêmes éditeurs (exempl. à Paris, Bibliothèque nationale de France; à Munich, Bayerische Staatsbibliothek, etc.). Enfin, au sujet de la figure alchimique de Démogorgon (p. 1198, n. 7), il existe un article très complet de Sylvain Matton : «La figure de Démogorgon dans la littérature alchimique » (in D. Kahn et S. Matton, éd., Alchimie. Art, histoire et mythes, Paris/Milan, SEHA/Archè, 1995, coll. Textes et travaux de Chrysopœia, 1, p. 265-346). Bien faibles compléments que ceux-ci à un pareil ouvrage.

Didier KAHN 
The Correspondence of Michael Faraday. Vol. IV : January 1849-October 1855. Letters 2146-3032. Ed. by Frank A. J. L. JAMES. Londres, The Institution of Electrical Engineers, 1999. 14,8 ×22,9, LXV- 1003 p., bibliogr., index.

Avec une moyenne d'environ 150 lettres par année, ce quatrième tome de la correspondance de Michael Faraday couvre une période où Faraday, après ses importantes découvertes dans les domaines de la chimie, de l'électricité et du magnétisme, est devenu la grande célébrité de la science anglaise au milieu du $\mathrm{XIX}^{\mathrm{e}}$ siècle. Le volume contient 887 lettres, y compris 6 lettres adressées à son épouse Sarah par des tiers, et 1 lettre de Sarah Faraday à John Barlow. 595 lettres sont publiées ici pour la première fois.

Comme dans les volumes précédents, les lettres sont présentées chronologiquement. Par conséquent, la correspondance scientifique avec les grands savants de l'époque, comme Airy (43 lettres), Tyndall (36 lettres), Plücker (20 lettres), de la Rive (20 lettres) et Schönbein (30 lettres), est entremêlée de lettres personnelles et de courts billets de circonstance (déclin d'une invitation, accusé de réception, etc.), ainsi que de documents qui sortent du cadre d'une correspondance au sens strict, comme, par exemple, un courrier de lecteur déplorant la pollution de la Tamise, publié dans le Times en juillet 1855, et un rapport de huit pages sur l'utilisation de la lumière électrique dans les phares.

Plusieurs lettres en provenance du continent démontrent que l'autorité de Faraday était également reconnue dans des pays éloignés de l'Angleterre. Lorsqu'en 1850, deux anciennes colonnes à Rome sont frappées par la foudre, Paolo Volpicelli, secrétaire de l'Académie pontificale des sciences, demande à Faraday (en français) « de vouloir bien exprimer son opinion très-appréciable » sur les possibilités de protéger ces monuments par des paratonnerres. Nous ne connaissons pas la réponse de Faraday, mais il semble que l'Académie l'ait appréciée car cinq mois plus tard, elle le nomme correspondant étranger.

Comme il serait vain de vouloir résumer tous les sujets abordés dans les lettres de ce volume, nous ne retiendrons que deux thèmes bien caractéristiques de la personnalité de Faraday : d'une part, ses attaques contre le spiritisme et les expériences pseudo-scientifiques des tables tournantes et, d'autre part, ses préoccupations religieuses exprimées dans ses lettres à William Buchanan, ancien de la Sandemanian Church d'Édimbourg.

À part quelques lettres en allemand et en italien, le français, que Faraday lisait facilement, est la seule langue étrangère présente dans cette correspondance. C'est la langue utilisée par les correspondants francophones comme Arthur-Auguste de la Rive, Antoine et Edmond Becquerel, Jean-Baptiste Dumas, Adolphe Quételet et Joseph-Antoine-Ferdinand Plateau, ainsi que par certains italiens comme Macedonio Melloni et Carlo Matteucci. Toutes ces lettres sont accompagnées d'une traduction anglaise.

Dans une introduction pertinente de vingt-deux pages, l'éditeur situe les lettres dans leur contexte scientifique, historique et biographique. En revanche, il y a peu de commentaires dans les notes où James s'est essentiellement borné à l'identification des personnages et des publications mentionnées dans la correspondance. Toutes 
ces publications sont regroupées dans la bibliographie. Dans un « Biographical Register », on trouve des informations biographiques sur les personnages qui apparaissent plus de deux fois dans le texte.

Le seul reproche que l'on puisse faire à cette édition est l'absence d'une table des correspondants où seraient indiquées les lettres respectives avec leurs dates. Le volume ne possède qu'un seul index pour les sujets et les noms des personnes, y compris ceux des correspondants. Si l'on ne s'intéresse qu'à la correspondance de Faraday avec un personnage précis, il faut donc d'abord chercher dans l'index le nom de cette personne, puis les mentions « Faraday writes to » et « Writes to Faraday ». C'est là que sont indiqués les numéros dont on a besoin pour trouver les lettres en question dans la correspondance. Cela est assez fastidieux, et il serait souhaitable qu'un index cumulatif de tous les correspondants, avec l'indication des lettres qu'ils ont expédiées et reçues, vienne compléter le dernier volume de ce corpus qui, d'ores et déjà, constitue un des grands monuments parmi les éditions de correspondances scientifiques indispensables à la recherche en histoire des sciences.

Andreas KLEINERT

Margherita Platania, éd., Les Mots de l'histoire. Le Vocabulaire historique du Centre international de synthèse. Naples, Bibliopolis, 2000. 15,5 ×23, 499 p. (Istituto Italiano per gli Studi Filosofici, ser. Testi, XIV).

Margherita Platania, Le Parole di Clio. Polemiche storiografiche in Francia. 1925-1945. Naples, Bibliopolis, 2001. 15,5 ×22,5, 132 p. (Istituto Italiano per gli Studi Filosofici, ser. Studi, XX).

Ainsi que l'explique l'auteur dans l'avant-propos - l'œuvre de grande ampleur qu'elle vient de produire en langue française chez un éditeur italien de Naples n'est qu'une simple esquisse de l'immense projet élaboré par Henri Berr d'un Vocabulaire historique, resté inachevé, et dont les inédits sont conservés, pour partie, dans les archives du Centre international de synthèse, et pour le reste, à l'Institut Mémoires de l'édition contemporaine (IMEC). Certains articles préparatoires avaient été publiés dans les Bulletins ajoutés à la Revue de synthèse historique. À partir d'un projet de 850 mots, seulement 250 ont atteint une forme quasi définitive ; Margherita Platania n'en présente que 27 accompagnés du compte rendu de la séance qui concernait individuellement chaque article.

Il faut remonter à 1925, année durant laquelle Berr annonçait la naissance du Centre international de synthèse avec la tâche de répondre à la crise contemporaine de la science. Berr voulait rendre à «l'effort des savants sa pleine valeur spéculative et morale » en développant l'esprit de synthèse, aussi bien dans chacune des sciences que dans l'ensemble des sciences. D'ailleurs, analyse et synthèse devaient renvoyer l'une à l'autre puisque l'une sans l'autre était soit stérile soit chimérique. 
Jusque-là avait régné sur l'Histoire le modèle positiviste, une discipline faite de documents et de textes, et privée d'hypothèses ou même de programmes de recherche. $\mathrm{Au}$ milieu propre aux historiens positivistes faisait face, derrière Émile Durkheim, celui des sociologues positivistes cherchant les lois de l'évolution humaine au-delà du « résidu » de l'événement. Certes, des innovations caractérisaient le paysage sociologique et, plus généralement, le paysage des sciences humaines dans lequel était née en 1900 la Revue de synthèse historique. Celle-ci avait eu pour mission de contrer l'abus de l'analyse et de l'érudition, la spécialisation et surtout la séparation entre les disciplines. La Revue avait introduit une « diversité qualitative par rapport aux vieilles thématiques "positivistes" sur la base desquelles la théorie et la pratique du temps se sont dégagées »(Les Mots de l'histoire, p. 23-24). L'Histoire des Langlois et Seignobos pouvait enfin être dépassée par une histoire consciente de ses méthodes et de ses hypothèses.

D'abord conçu comme un moment de recherche historique, le Centre devait en outre « fournir au citoyen les moyens de s'approprier l'outillage mental de la civilisation moderne » (Giuliana Gemelli, cité p. 27). Platania nous fait donc pénétrer dans la complexité du débat épistémologique tel qu'il fut vécu à l'époque ; le Vocabulaire historique appartient à cette polémique : l'état d'inachèvement dans lequel il est resté en est aussi un témoignage manifeste. Six Commissions organisées avec les animateurs adéquats avaient eu pour charge de « définir rigoureusement les termes dont se servent les historiens et fixer, autant que possible, les notions fondamentales de leur science, en donnant l'état des problèmes théoriques et la bibliographie correspondante » (Berr, cité p. 31). Le dessein originaire de 400 mots avait peu à peu été porté à plus de 800. De l'analyse des mots choisis au cours du temps, allant de 1925 à 1945, Platania a su tirer des conclusions ponctuelles fort intéressantes. En effet, de l'examen de l'élaboration de quelques-unes des notions, il ressort que les rédacteurs du Vocabulaire historique n'étaient pas nécessairement d'accord avec leur directeur de publication. Comme l'écrit Platania, dans ce vocabulaire, « on trouve [...] des idées dépassées et des perspectives nouvelles, des polémiques récentes mais aussi d'anciens contrastes » (p. 375).

Parmi les «mots de l'Histoire », il peut être intéressant de s'attarder un instant sur le mot « causalité » étudié par Gaston Bachelard et sur la discussion qui suivit, concernant la notion. Ce qui n'est en fait qu'une note peu développée de la part de Bachelard montre que «la causalité pose des problèmes de structure » (p. 147). Contre l'évidence de la cause et l'unité de temps, Bachelard proposait plutôt l'idée de fonction, plus intéressante pour le physicien, ainsi que des temps multiples, même si, pour lui, le « vieux causalisme » avait du mal à «s'en aller» (p. 148). D'ailleurs, Bachelard fait de la cause une «métaphore » et, du temps, un « temps postiche ». La séance du 17 mai 1944 sur la causalité est assez étonnante : elle illustre la position des intervenants selon laquelle le concept de causalité aurait été définitivement périmé. Berr, au contraire, défend les idées de causalité logique et de causalité légale, qu'il juge nécessaires en histoire ; tout comme, en 1926, la causalité en général avait été considérée comme nécessaire en sociologie par Daniel Warnotte, de l'institut Solvay à Bruxelles, auquel Berr s'était adressé pour éviter les controverses françaises courantes entre historiens et sociologues ; celui-ci 
définit la sociologie comme « la science qui se propose d'expliquer les relations de cause à effet réglant les interactions auxquelles sont soumis les hommes qui vivent en société » (p. 465).

Angèle KREMER MARIETTI

Lectures de Michel Foucault. Vol. II : Foucault et la philosophie. Textes réunis et introd. par Emmanuel DA SILVA. Lyon, ENS Éditions, 2003. 14,7×20,7, 136 p. (Theoria).

Lectures de Michel Foucault. Vol. III : Sur les Dits et écrits. Textes réunis et introd. par Pierre-François MOREAU. Lyon, ENS Éditions, 2003. 14,7 × 20,7, 104 p. (Theoria).

Dans les lignes qui suivent, les abréviations suivantes sont utilisées :

$H F$ : Histoire de la folie (1961)

$N C$ : Naissance de la clinique (1963)

RR : Raymond Roussel (1963)

$M C:$ Les Mots et les choses (1966)

AS : L’Archéologie du savoir (1969)

SP : Surveiller et punir (1975)

DE : Dits et écrits (1994)

Presque vingt ans après la mort de Michel Foucault, son œuvre est l'occasion de réflexions renouvelées. La première vague d'émotions passée, c'est le penseur qui triomphe de l'homme. Plusieurs constats peuvent être faits, communs à ces deux tomes édités par l'ENS des lettres et sciences humaines (après celui consacré à Il faut défendre la société). La première livraison (vol. II), présentée par Emmanuel Da Silva, s'intéresse tout autant à l'œuvre de Foucault comme philosophie et à la place singulière que celui-ci y occupe désormais, qu'au rapport qu'il entretient avec la tradition philosophique (implicitement ou explicitement). Si seul le volume III est entièrement consacré aux $D E$, ce qui frappe rétrospectivement, c'est partout le recours massif à ces textes précieux qui ont indiscutablement modifié notre approche de Foucault : qui l'ont affinée, approfondie, complexifiée. Entre ce que Pierre-François Moreau nomme « les grands livres » et les cours au Collège de France, l'ensemble des $D E$ assume un rôle singulier, irréductible aux deux autres ensembles et marqué par des traits spécifiques qu'il recense synthétiquement. En résultent des approches moins polémiques et de frappantes convergences qui tracent, pour les deux volumes, et comme indépendamment de leurs thématiques spécifiques, des lignes transversales, soit sur le plan des contenus, soit sur le plan de l'approche. Particulièrement récurrents, les thèmes du sujet, de l'expérience ou de l'éthos à travers les derniers textes du « dernier » Foucault, les articles semblant parfois s'interpeller, se compléter et débattre. Véritable leitmotiv de ces pages, le 
commentaire du Qu'est-ce que les Lumières? de Kant motive de multiples questions et nourrit nombre d'approches.

Vol. II : Foucault et la philosophie.

Jacques D'Hondt ouvre ce volume de manière polémique en interrogeant «l'épistémologie discontinuiste » de Foucault. Il remet d'abord en perspective cette idée, qui, de Blaise Pascal à Claude Bernard, fait de l'histoire le terrain d'un progrès continu et croissant des connaissances humaines. S'appuyant sur la notion d'épistémè, il situe Foucault, a contrario, dans une tradition récente qui remonte à Bachelard, transite par Althusser, et dont le dénominateur commun serait le refus de toute filiation. Contre cette «idéologie de la rupture », qui fait de l'histoire une simple «projection de diapositives », l'auteur réaffirme un continuisme indépassable : toute rupture, de fait, renvoyant toujours secrètement à une continuité. Foucault apparaît donc tout à la fois comme une victime et un propagateur aveugle de ce « rupturalisme ». À son insu, c'est finalement en continuateur paradoxal de l'idéalisme transcendantal et, au-delà, de la philosophia perennis qu' apparaît ici Foucault...

Béatrice Han explore, quant à elle, la notion d' « a priori historique ». La notion marque la démarche du premier Foucault pour disparaître ensuite, mais son enjeu, essentiel, est de poser la question des conditions de possibilité du savoir. Elle va se déployer progressivement à travers les déplacements et les redéfinitions d'un concept évolutif, finalement inadéquat à son objet et doublement problématique : Comment un a priori peut-il être « historique »-ce qui implique sa concrétude, son empiricité ? Comment peut-il à la fois manifester son enracinement husserlien, tout en s'en démarquant sur l'essentiel - l'a priori historique n'étant pas un universel ? Foucault laissera planer cette indétermination conceptuelle jusqu'à l'AS, première réelle tentative pour fonder philosophiquement la notion. La $N C$, les $M C$ et l'AS élaboreront trois définitions différentes, témoignant indissolublement d'une tentative de thématisation et d'une sorte de fuite en avant théorique. D'un enracinement marqué par la phénoménologie de Maurice Merleau-Ponty - sa terminologie plus que sa problématique - la notion évolue dans la $N C$ vers une « ontologie réaliste » d'inspiration aristotélicienne ; l'AS, enfin, éradiquera cette métaphysique inavouée et encombrante, par un recours durable au nominalisme, l' « a priori historique » n'étant plus alors conçu qu'à travers la «fonction énonciative ». L'auteur débusque, toutefois, une circularité révélatrice de l'impasse dans laquelle est engagé Foucault et qui invalide le concept lui-même plus que la démarche archéologique. En témoignent tout à la fois son effacement après l'AS et la résurgence du problème qu'il posait, à travers de nouveaux déplacements.

Les philosophes que j'aime, disait Foucault, je ne les cite pas, je les utilise : le rapport à Spinoza, exploré ici par Olivier Remaud, pourrait en être un excellent exemple. Si l'on peut souscrire à sa thèse d'un spinozisme radical de Foucault, force est de reconnaître que l'auteur de l'Éthique n'est pratiquement jamais mentionné. Ce ne sont pas tant les thèmes qui rapprochent les deux philosophes qu'une certaine démarche qui lie philosophie et politique ainsi qu'une certaine identité du questionnement. Ainsi, sur la question centrale du pouvoir, tous deux considèrent qu'il n'existe qu'en acte, n'a pas d'essence propre et réside dans un 
ensemble de relations rétives à l'analyse juridique. Mais, c'est sur la notion d'affect que l'auteur va concentrer ses analyses, montrant que toute la question de la «gouvernementalité » est sous-tendue par elle. C'est désormais à travers les analyses du Foucault de Gilles Deleuze (Paris, Minuit, 1986) que l'auteur va aborder la question du rapport à Spinoza, lui permettant de lever des difficultés proprement méthodologiques soulevées par son approche initiale. Deleuze, explique-t-il, propose un « coup de force » en réinterprétant radicalement Foucault en termes spinozistes. Or, ce détour va lui permettre de suturer les analyses du pouvoir et l'éthique de la subjectivité, souvent décrétées incompatibles. C'est, pour finir, une problématique d'une très grande cohérence qui se dessine à travers cette grille de lecture, permettant, tout comme chez Spinoza lui-même, de réconcilier liberté et nécessité à l'intérieur d'une éthique, dévoilant chez Foucault une « véritable pensée de l'immanence ».

C'est encore la question du rapport à Deleuze, envisagée cette fois frontalement, qui motive l'étude de Didier Ottaviani, à travers le passage de la société disciplinaire à la société de contrôle. Après un rappel des analyses foucaldiennes de la discipline, l'auteur explore la société de contrôle à travers les analyses deleuziennes, la société disciplinaire n'étant que ce socle, aujourd'hui dépassé, à partir duquel la société de contrôle a vu se profiler ses inquiétants contours. L'auteur va alors développer très librement des aperçus sur la société contemporaine, mettant en œuvre les analyses et la terminologie deleuziennes (flux, segmentation, territorialisation/ déterritorialisation, agencement, espace lisse/espace strié, etc.). Même si l'auteur prend soin de préciser que Foucault lui-même a indiqué ce mouvement qui conduit des disciplines au contrôle, il reste que la belle rigueur foucaldienne se voit progressivement happée par la prose imagée mais parfois bien nébuleuse de Deleuze...

Relisant SP, l'historien Philippe Artières étudie la prolifération des pratiques scripturaires personnelles en prison. Le supplice, la punition et la discipline distinguent trois techniques politiques du corps, mais aussi trois strates historiques, trois pratiques pénales distinctes. Or, à ces trois strates correspondent aussi trois statuts de l'écrit. De manières bien différentes, la pénalité des supplices et celle des réformateurs confisquent l'écriture au profit du seul pouvoir (glorification de l'autorité royale ou édification morale et civique). L'ère carcérale va déplacer le pôle de l'écriture qui n'est plus directement celle du pouvoir mais sollicite le détenu lui-même : «[...] la prison va créer de toutes pièces un scripteur et avec lui tout un corpus. Ce scripteur sera le délinquant, ce corpus, la littérature des prisons » (p. 83). Sous des formes variées, l'écriture du délinquant va se déployer : autobiographies de criminels, dossiers divers, tatouages, etc. On comprend mieux alors l'étrange expression de «panoptique graphique » qui intitule ce texte, comme si l'écriture «personnelle » redoublait l'enfermement et recréait, à sa manière, la diabolique machine de Bentham.

C'est le rapport de Foucault à la Renaissance qu'examine Tristan Dagron, à travers une lecture de deux textes qui se répondent, comme l'histoire de l'Autre à celle du Même, l'HF et les $M C$, et que l'auteur va entrecroiser savamment. À travers un parcours sinueux, complexe et très informé, l'auteur montre que la lecture qu'a faite Foucault de la Renaissance est cohérente, originale et productive. 
Entre l'approche de la folie et les analyses de l'épistémè du XVI ${ }^{\mathrm{e}}$ siècle se tissent alors des liens puissants et inattendus. Contre le jugement critique de certains spécialistes, il souligne que leur lecture, trop rapide, s'en tient à l'opposition factice entre « ressemblance » et « représentation » et ne résiste pas à l'analyse serrée de Foucault dont l'intérêt est ailleurs. L'auteur invoque alors le témoignage de philosophes, s'appuyant sur la notion de « sympathie » pour conforter l'interprétation de Foucault dont le mérite est finalement d'avoir su décrypter la Renaissance sans y interposer de schéma préalable : « Il ne s'agit pas de repérer ce qui, à la Renaissance, préfigure les systèmes de l'âge classique, mais tout au contraire, de comprendre la synthèse à partir d'un point de vue qui lui échappe » (p. 113).

Franck Fischbach explore, pour finir, le commentaire, par Foucault, du Qu'est-ce que les Lumières? de Kant, objet de son étonnement sur plusieurs plans. Foucault a entendu inscrire son travail dans le sillage du Kant de l'Aufklärung et de l'école de Francfort, or celle-ci, souligne l'auteur, a eu un rapport très critique à l'Aufklärung. Un double axe d'exploration est alors retenu : la question du présent et la critique de l'Aufklärung ; mais c'est chez Hegel, et non chez Kant, que les deux se concilient et c'est seulement à partir de Hegel, véritable penseur de la modernité, comme l'a souligné Jürgen Habermas, qu'il est cohérent de poser la question de la postérité de l'Aufklärung. En fait, une double distance semble manquer à Kant : un recul strictement temporel et un recul affectif. Or, souligne l'auteur, seuls les post-kantiens pourront prétendre avoir cette distance pour «diagnostiquer» le présent. Ce qui est reproché ici à Foucault, c'est donc une lecture anachronique de Kant qui contourne et relativise sciemment Hegel tout en «hégélianisant» sa lecture de Kant, Hegel apparaissant alors comme la vérité ultime et de Kant et de Foucault. Ce dernier serait donc à la fois dans et hors de l'Aufklärung : dehors par la dimension critique qu'il entend maintenir, dedans par fidélité à l'attitude kantienne elle-même, paradoxe dont le lieu de résolution pourrait être cet «ethos philosophique »si déterminant pour le dernier Foucault.

\section{Vol. III : Sur les Dits et écrits.}

C'est dans les années allant de 1954 à 1969 que prolifèrent les textes de Foucault sur la littérature, trahissant un véritable champ d'attraction et de préoccupations spécifiques sur les objets les plus divers. Or ces textes, remarque Philippe Sabot, semblent s'épanouir à la marge de l'œuvre : en dehors du $R R$, en effet, aucun livre n'est consacré à l'œuvre d'un écrivain précis, même si de précieux contrepoints littéraires émaillent l' $H F$ ou les $M C$. Or, cet étrange corpus, sans réelle unité, entretient avec l'œuvre livresque des liens féconds et ténus d'implication réciproque, dévoilant une vérité essentielle que la réflexion archéologique ne peut prendre en compte. La littérature, et elle seule, témoignera de «l'expérience de la négativité » d'une folie dont les raisons apparaissent ailleurs. C'est parce que la littérature explore une limite que son précieux témoignage est requis depuis ce non-lieu que constituent les $D E$. Ainsi ces « expériences radicales de langage » (Sade, Hölderlin, Artaud ou Bataille), expériences de la transgression, ouvrent une dimension irréductible à tout discours théorique, historique ou même archéologique, dont elles apparaissent comme le nécessaire contrepoint : d'où l'importance particulière 
de l'œuvre de Raymond Roussel. Ce n'est pas le cas psychiatrique de Roussel qui intéresse Foucault, mais bien, à travers l'écriture, cette « expérience radicale de la finitude » qui met en scène le vertige de la mort quand, dans le même temps, la $N C$, écrite la même année, s'attache à en cerner les contours objectifs. Ainsi, loin d'éloigner de la philosophie, la littérature, à travers ces expériences de la limite, y reconduit. Un pont peut même alors être jeté vers le dernier Foucault de «l'éthique de la subjectivité » et de «l'esthétique de l'existence».

Il est d'usage d'opposer le Foucault penseur du pouvoir à celui de l'éthique de la subjectivité. D'entrée de jeu, Jean-François Pradeau montre qu'il n'y a jamais eu deux statuts antithétiques du sujet, comme on s'est trop plu à le dire, cette ancienne préoccupation émergeant très tôt à l'intérieur d'une problématique des relations de pouvoir. De fait, la notion clé d' " assujettissement » est incompréhensible sans la référence à ce sujet qui se constitue dans et par elles. S'attardant particulièrement sur le commentaire de l'Alcibiade de Platon et sur la notion de parrêsia, comme expérience subjective de la vérité, Pradeau montre qu'éthique et politique ne sont pas antithétiques, mais complémentaires. Une «éthique de la subjectivation », plutôt qu'une morale normative fondée sur un sujet transcendant, portera l'accent, non sur le sujet constitué, mais sur les processus qui transforment l'individu en sujet. La valorisation de l'expérience des Grecs anciens aura précisément pour mission de montrer qu'il n'y a pas lieu d'opposer esthétique, morale et politique. L'auteur revient alors sur le projet de Foucault, jamais rempli, d'une recherche sur le «Gouvernement de soi et des autres », qui aurait dû articuler l'éthique sur la politique via les analyses de la « gouvernementalité ». Si ce projet n'est pas rempli, poursuit-il, c'est moins en raison de la mort brutale du philosophe qu'en raison d'une tension non maîtrisée entre deux lignes argumentatives qui ne coüncident pas : le pôle éthique et celui de la subjectivité restent disjoints. C'est, pour finir, un double statut de la vérité qui apparaît, comme si Foucault n'avait pas su choisir entre une vérité de l'affirmation subjective et l'analyse objective des procédures politiques que sous-tendait la notion de « gouvernementalité ».

À partir des $D E$, Bernard Vandewalle recense cinq éléments constitutifs du dispositif anthropologique de Foucault, distincts et combinables, qu'il explore successivement : l'existence d'une limite qui ne fait qu'un avec l'existence humaine ; l'absence d'une vérité originaire ; le fait que le savoir humain ne peut se constituer que dans l'évanouissement de son objet ; la prééminence des procédures disciplinaires dans la formation des savoirs ; enfin, l'obstacle récurrent d'une nature humaine : tout projet archéologique impliquera, en ce sens, une critique préalable de l'illusion anthropologique. En ce bref et suggestif parcours d'éléments au premier abord disparates, l'auteur finit par dégager une grande cohérence : ce qui motive Foucault, ce n'est pas la question kantienne «Qu'est-ce que l'homme?», mais l'analyse des conditions de possibilité de cette question. L'homme n'est, pour finir, qu' un « indicateur épistémologique », une fonction qui se noue dans la relation du savoir et du pouvoir. La sortie du sommeil anthropologique, pourtant, à son tour, n'est peut-être qu'une illusion. Seul recours: le patient mais interminable travail d'historicisation des thèmes étudiés... 
À travers un parcours sinueux mais précis, Adolfo Marino s'efforce de renseigner et de confronter longuement les références foucaldiennes à Platon, à Kant, à Heidegger, au risque parfois de perdre de vue la démarche qu'il est censé éclairer. Trois axes sont retenus : le rapport subjectivité/vérité, la conception d'une subjectivité non introspective et l'élaboration d'un « ethos philosophique ». Le problème est alors de comprendre comment s'articulent l'historicité d'un sujet assujetti et la nécessaire prise en compte d'une autonomie, même relative, de ce sujet. Or, le lieu de cette articulation, ne peut être que la marge : «Le souci de soi est une pratique de la frontière. » Lutte contre soi-même, le souci de soi débouche sur une attitude limite définissant la modernité. Foucault retrouve alors les trois grandes Critiques kantiennes et les questions qui y correspondent et semble réactualiser un texte ancien dont on retrouve la valeur programmatique : sa thèse complémentaire sur l'Anthropologie de Kant.

C'est à l'étrange rapport qui lie Kant à Baudelaire dans les deux versions (américaine et française) du commentaire de Foucault du Qu'est-ce que les Lumières ? de Kant, que s'intéresse Fabienne Brugère. Elle met alors en évidence trois dimensions du commentaire de Foucault : la dimension politique, inséparable de ce risque qu'il y a à penser le présent, la dimension philosophique qui rend notre actualité solidaire de cette épistémè inaugurée par Kant et la dimension éthique, enfin, qui débouche sur une attitude : un ethos qui s'ouvre sur la liberté et se caractérise par un franchissement. Mais la modernité recèle encore une dimension proprement esthétique, à la fois interne et externe à la pratique artistique que Baudelaire, comme créateur et comme théoricien de sa propre pratique, incarne remarquablement. Toutefois, l'attitude esthétique de Baudelaire ne recoupe pas exactement l'attitude philosophique de Kant : décalée vers l'esthétique de l'existence à travers la figure du dandy, elle semble faire passer au second plan la question de l'ontologie $d u$ présent qui l'avait pourtant requise. Le compositeur Pierre Boulez pourrait alors apparaître comme un représentant plus authentique de cette attitude dans laquelle pensée, transgression et création se trouvent confondues.

Dans le dernier article, Frédéric Gros interroge efficacement l'étrange et persistante manière dont Foucault s'est, tout au long de sa carrière, approprié son œuvre, et singulièrement dans ces éléments extérieurs que constituent les $D E$. Ce qui frappe dans la stratégie de Foucault, c'est la très grande plasticité de ses positions, au point que les différentes redéfinitions proposées peuvent alors apparaître comme autant de contradictions. Or, Foucault a toujours insisté, jusqu'à l'improbable parfois, sur l'unité de sa démarche. Rejetant tout à la fois les pistes faciles de l'idée polémique d'une coquetterie de l'auteur et celle d'une hiérarchie discriminante entre les $D E$ et les œuvres patentées, Gros montre dans toute sa complexité la position de Foucault, position d'autant moins cernable qu'elle se différencie assez nettement selon les époques. Il met en évidence plutôt une sorte d'enveloppement : revisitant ses textes anciens, Foucault y projetterait son livre futur. À travers l'idée de l'œuvre comme fiction, puis comme expérience, dans laquelle 
l'auteur, comme le lecteur, sont appelés à se modifier, Foucault finit pourtant par aboutir, dans ses tout derniers textes, à rendre crédible et cohérente une unité jusqu'ici plus affirmée que réelle, à travers l'axe Savoir/Pouvoir/Sujet, qui s'opère autour des analyses du Qu'est-ce que les Lumières? de Kant.

François BoullaNT 